\title{
Domain-driven Discovery of Stable Abstractions for Pointcut Interfaces
}

\author{
Dimitri Van Landuyt, Steven Op de beeck, \\ Eddy Truyen and Wouter Joosen \\ DistriNet, Katholieke Universiteit Leuven \\ Celestijnenlaan 200A \\ B-3001 Leuven, Belgium \\ \{dimitri.vanlanduyt,steven.opdebeeck, \\ eddy.truyen,wouter.joosen\}@cs.kuleuven.be
}

\begin{abstract}
The benefits of defining explicit pointcut interfaces in aspectoriented applications have been advocated by many. A pointcut interface exposes a set of crosscutting abstract behaviours (as named pointcut signatures) that multiple aspects in the application can use. In accordance with the dependency inversion and stable dependencies principles, a pointcut interface should expose only stable abstractions in order to maximally promote its reuse across a family of applications.

In this paper, we propose a domain-driven architecture method for designing such stable pointcut interfaces. The method employs systematic reengineering of use case models to discover stable abstractions that are anchored in the domain model of the application. During architecture design, these stable domain abstractions are mapped to pointcut interfaces. As part of this mapping activity, the architecture is constrained to ensure that the pointcut interfaces can be implemented correctly.

We have applied this method in two applications, where we validate that pointcut interfaces can be reused for implementing the composition logic of different aspects without requiring modification to their pointcut signatures. Moreover, the method consistently yields pointcut interface hierarchies.
\end{abstract}

\section{Categories and Subject Descriptors}

D.2.13 [Software Engineering]: Reusable Software-Domain engineering; D.2.11 [Software Engineering]: Software architectures-Data abstraction, Domain-driven architecture

\section{General Terms}

Design, Documentation

\section{Keywords}

Domain-driven Architecture, Reusable Pointcut Interfaces, Stable Domain Abstractions

Permission to make digital or hard copies of all or part of this work for personal or classroom use is granted without fee provided that copies are not made or distributed for profit or commercial advantage and that copies bear this notice and the full citation on the first page. To copy otherwise, to republish, to post on servers or to redistribute to lists, requires prior specific permission and/or a fee.

AOSD'09, March 2-6, 2009, Charlottesville, Virginia, USA

Copyright 2009 ACM 978-1-60558-442-3/09/03 ...\$5.00.

\section{INTRODUCTION}

Aspect-oriented programming enables the modularization and composition of crosscutting concerns. However, in large-scale applications the necessary composition logic remains often complex and it would greatly pay off if the composition logic traditionally encoded in monolithic pointcut specifications- could be reused within a single application or across a family of applications. Monolithic pointcuts are often written with a specific aspect composition in mind and are highly dependent on low-level details of the application. This causes any form of reuse to be problematic at best.

The stable dependencies principle (SDP) and dependency inversion principle (DIP) applied to aspects [37, 14] state that, in order to maximally promote pointcut reuse, (a) a pointcut should only depend on abstractions, and (b) the more pervasive a pointcut becomes, the more it should depend on abstractions.

Two mechanisms are essential for effective pointcut reuse: (i) named pointcuts, that is, supplying a pointcut with a suitable name that describes its intention or meaning; and (ii) abstraction, in the sense of key abstractions that reflect concepts in designers' minds and communication. Putting both together results in pointcuts that carry the name of the key abstractions they represent. This enables two-level pointcut design where named pointcuts are used by a composition pointcut that binds an aspect to the application. Figure 1 presents the structure of this two-level approach and illustrates the different types of pointcut reuse that should be supported.

This idea is not new, its essence has been covered by, for example, the pointcut interface from the work of Gudmundson, et al. [12] and as a part of the crosscutting programming interface (XPI) from the work of Griswold, et al. [34]. To summarize Gudmundson [12]: a pointcut interface is an interface between the base and the aspects that are applied to it. This interface contains a number of pointcut definitions that represent abstraction points in the base. A pointcut interface is maintained together with the module that it refers to. To support this idea at the architectural level, Kulesza, et al. [19] introduce extension join points (EJPs) which establish a contract between the base and aspectual modules.

Aspect-oriented programming languages provide us with a way to capture abstractions as named pointcut signatures (a name and a set of parameters), which are logically grouped into pointcut interfaces. However, no guidelines are available on how to identify the abstractions that lead to reusable pointcut signatures - Section 6 gives an elaborate account about this lack of guidance. Most work on this topic is based on a bottom-up approach, where a useful pointcut is defined in the scope of a specific aspect and is given a name that best captures its meaning in that specific scope. 
Moreover, the parameters of the pointcut signature are typically designed with that single aspect in mind. The pointcut can often not be reused for another aspect.

We therefore explore and study the value of a top-down approach that is based on an architecture creation process, during which all known and relevant crosscutting concerns are reasoned about. Moreover, as a second guiding principle, we expect that the stability of the pointcut signatures will considerably improve if their definition is based on stable domain abstractions. Stable domain abstractions are fundamental concepts in the application domain that change slowly [32].

The main contribution of this paper is twofold: (1) We introduce a method for the discovery of domain abstractions by systematically reengineering use cases. (2) We define a method for the mapping of these domain abstractions to reusable pointcut interfaces at the level of architecture. As part of this mapping activity, the architecture is constrained to ensure that the pointcut interfaces can be implemented correctly.

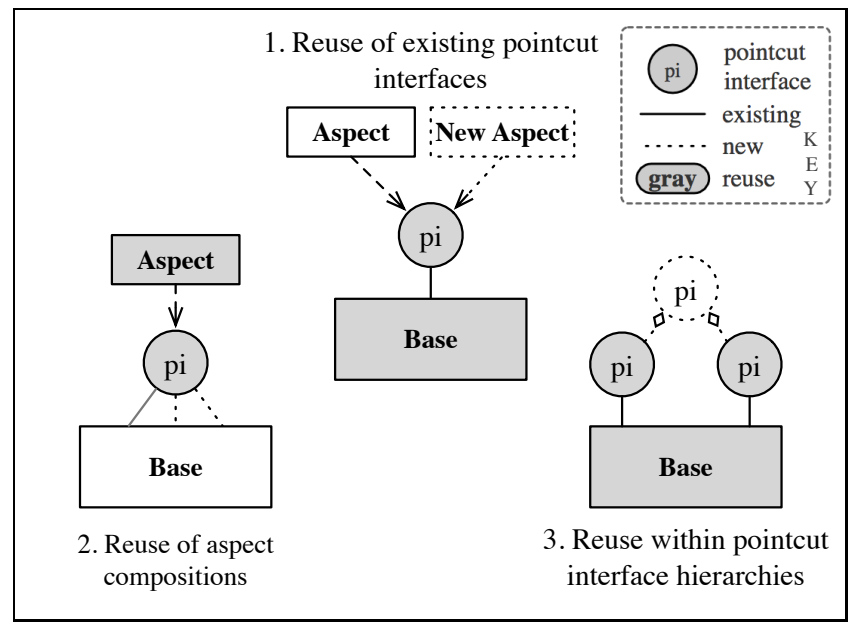

Figure 1: Types of pointcut reuse.

Additionally, we have applied the proposed method in two applications, where we validate that pointcut interfaces can indeed be reused for implementing the composition logic of different aspects without requiring modification to their pointcut signatures. To this end, we distinguish between three types of pointcut interface reuse (as illustrated in Figure 1):

1. Reuse of existing pointcut interfaces. This type of reuse occurs when a new aspect is introduced that can be composed with the application in terms of already-existing pointcut interfaces, either fully or partially. Diagram 1 in Figure 1 illustrates this type of reuse graphically.

2. Reuse of aspect compositions. This type of reuse occurs when a new requirement is added that affects an already-existing crosscutting concern. Reuse is successful when the new requirement can be supported by re-implementing an existing pointcut interface, without breaking the pointcut signatures. The reimplementation typically involves expanding the pointcuts to a larger join point set in the base application. This type of reuse is illustrated in diagram 2 of Figure 1.

3. Reuse within pointcut interface hierarchies. This type of reuse occurs when the pointcut interfaces themselves are structured hierarchically; i.e. the implementation of high-level pointcut interfaces is done in terms of lower-level ones. This structuring avoids unnecessary duplication of pointcut interface specifications, and whenever the implementation of a lower-level pointcut changes, the higher-level pointcut reuses this change at no extra cost. Figure 1 illustrates this type of reuse in diagram 3.

Our domain-driven architecture method is situated at the transition between requirements elicitation and architecture creation. This work is related to various Early Aspects [4] approaches that map aspectual requirements to architecture. The most relevant approaches in this space include COMPASS [7], Goal-Driven Discovery of Aspects [27], Theme [8], Jacobson's AOSD with Use Cases approach [15] and Aspect-Oriented Architecture Models (AAM) [10]. The main and common goal of all the above Early Aspects approaches is achieving improved aspect traceability across the software life cycle and supporting trade-off analysis [30, 27] for conflicting requirements. The proposed architecture method is complementary to these approaches as we focus on the discovery of stable abstractions for aspect-oriented architecture and composition, starting from the requirements.

The remainder of the paper is structured as follows. Section 2 introduces the starting points for this paper, whereas Section 3 presents a high-level overview of the proposed method. In Section 4 , a detailed application of the method is illustrated by means of an example from one of the case studies. Then, Section 5 summarizes our findings with respect to the actual reusability of the discovered pointcut interfaces, based on two case studies in which the approach has been applied. Related work is presented in Section 6. Finally, Section 7 concludes the paper and presents future work.

\section{STARTING POINTS}

In this section, we introduce the methodological starting points for our approach. Section 2.1 introduces stable domain abstractions and discusses domain stability. Section 2.2 discusses how use cases are engineered to improve separation of concerns. Finally, Section 2.3 introduces the Twin Peaks model, to which our approach adheres.

\subsection{Stable Domain Abstractions}

Sommerville [32] defines stable domain abstractions as "fundamental concepts in the application domain that change slowly". In a realistic development setting, the problem domain is often the only common ground between the different developers and therefore, stable domain abstractions form an important means of communication between the different members of the development team. In domain-driven design [9], the set of stable domain abstractions is called a ubiquitous vocabulary.

Additionally, the use of stable domain abstractions has been linked to effective reuse in literature [32, 24]; i.e. in a componentbased context, components implementing a stable domain abstraction are more likely to be reusable. Literature shows that for several often-recurring concerns, the search for stable domain abstractions has indeed converged. Such concerns are infrastructural in nature, such as transaction handling, caching and security. For instance, the EJB 3.0 standard introduces a set of annotations which are used to bind aspects to an application, and are reusable across applications.

In this paper, we propose an approach to discover stable domain abstractions, and leveraging their stability to build pointcut interfaces that are resilient to evolution. Therefore, the starting point is absolute domain stability; i.e. the situation where the domain itself remains fully stable when requirements change. Obviously, absolute domain stability is an unrealistic condition in real-world 


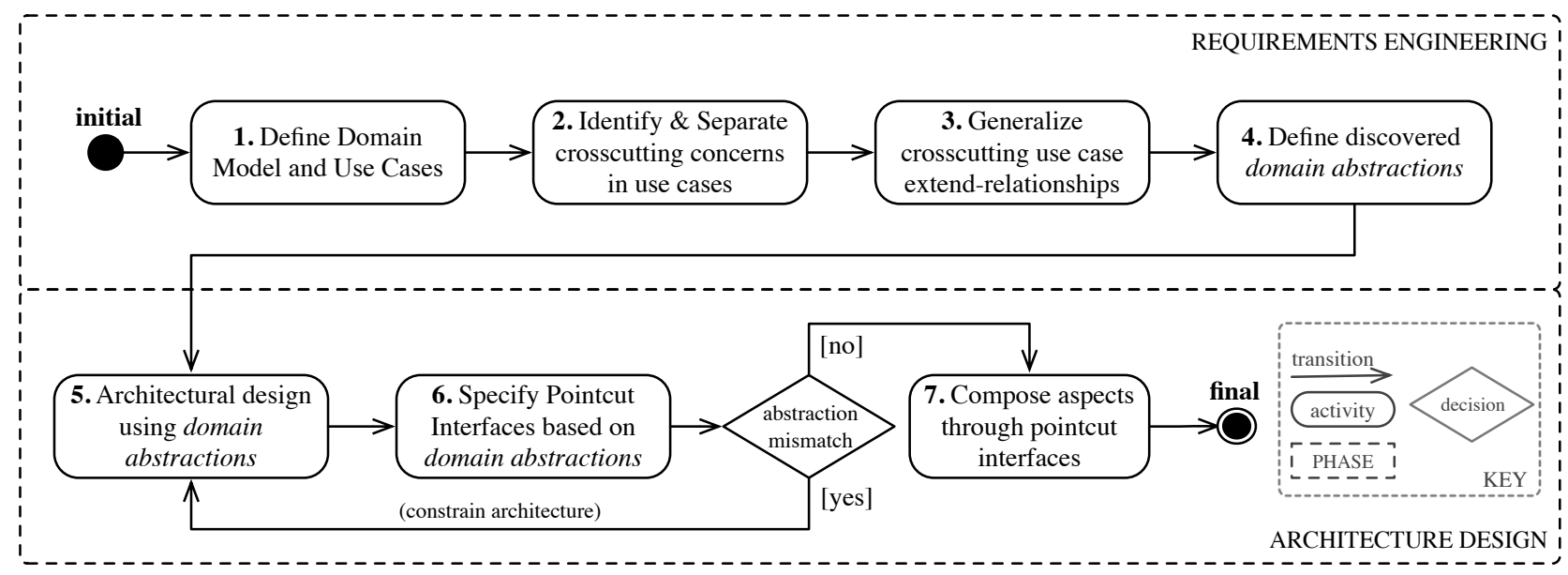

Figure 2: Overview of the method.

problem domains, as it is impossible to take into account all possible future changes in a software system. Section 5 investigates practical reuse in two realistic case studies, and their relation to domain stability. We show that reuse improves in any case, and that reuse seems to be more optimal in the context of a stable problem domain.

\subsection{Use Cases and Aspects}

We propose a use case-driven approach to find stable domain abstractions by reengineering use case diagrams.

Part of the approach deals with the separation of base use cases and extension use cases. Use case extension [15] is a use case relationship that is well-suited for this purpose. Introducing this relation requires explicitly naming extension points to be defined by the base use cases. One common pitfall in specifying extension points is naming them after the aspects that affect them. This approach inherently suffers from the problem of concern diffusion: the base is affected in a crosscutting manner by the aspects. Instead, extension points should be used to expose additional information specific to the base concern, regardless of any specific aspect. This is exactly what our architecture method advocates.

Our approach introduces a use case hierarchy, in which abstract use cases represent commonalities between use cases. However, the UML specification is not clear on the semantics of an extension point in an abstract use case. To avoid confusion, we attribute the following semantics to extension point inheritance: the abstract use case provides a common frame (segment of abstract steps) in which all child use cases must be structured. In this frame, an extension point is defined. Child use cases implementing this frame automatically inherit the extension point. This way, an extension point denotes a step that is common to all child use cases.

\subsection{Twin Peaks model}

Our architecture creation approach adheres to the Twin Peaks model to Software Engineering [28]. This model represents an incremental software development process which spirals between requirement analysis and architecture design. Developers alternatively iterate over system requirements and architecture design in quick succession, developing the application's requirements and architecture specifications concurrently. For reasons of simplicity, Figure 2 outlining the approach does not depict this explicitly. At any point in time, the software engineer might backtrack to one of the requirement activities.
Table 1: Input and output artifacts per activity.

\begin{tabular}{|c|l|l|}
\hline Act. & Input & Output \\
\hline 1 & $\begin{array}{l}\text { stakeholder requirements, } \\
\text { domain analysis, techni- } \\
\text { cal/legal constraints, .. }\end{array}$ & use cases, domain models \\
\hline 2 & use cases & $\begin{array}{l}\text { separated use cases; exten- } \\
\text { sion points }\end{array}$ \\
\hline 3 & $\begin{array}{l}\text { separated use cases; exten- } \\
\text { sion points }\end{array}$ & $\begin{array}{l}\text { generalized use cases; ab- } \\
\text { stract extension points }\end{array}$ \\
\hline 4 & $\begin{array}{l}\text { abstract extension points; } \\
\text { domain models }\end{array}$ & domain abstractions \\
\hline 5 & $\begin{array}{l}\text { use cases; domain mod- } \\
\text { els; domain abstractions; } \\
\text { existing component-based } \\
\text { architecture (optional) }\end{array}$ & $\begin{array}{l}\text { (constrained) component- } \\
\text { based architecture }\end{array}$ \\
\hline 6 & $\begin{array}{l}\text { component-based ar- } \\
\text { chitecture; } \\
\text { abstractions domain }\end{array}$ & $\begin{array}{l}\text { component-based ar- } \\
\text { chitecture with pointcut } \\
\text { interfaces }\end{array}$ \\
\hline 7 & $\begin{array}{l}\text { component-based ar- } \\
\text { chitecture with pointcut } \\
\text { interfaces }\end{array}$ & $\begin{array}{l}\text { component-based } \\
\text { chitecture with pointcut } \\
\text { interfaces and aspectual } \\
\text { compositions }\end{array}$ \\
\hline
\end{tabular}

\section{OVERVIEW OF THE METHOD}

This section briefly introduces the proposed approach. Figure 2 presents an activity diagram showing the sequence of steps to be performed. It depicts how the approach guides the software engineer throughout seven activities to discover and design an architecture that is centered around reusable pointcut interfaces. Additionally, Table 1 presents the input and output artifacts for each activity.

The approach shows similarities to the "AOSD with Use Cases Approach" by Jacobson [15]. In activity 1, the requirements are gathered and modeled in a structured way, as use cases. Additionally, as our approach is domain-driven, a domain model for the application is defined in parallel.

In activity 2 , the identification of crosscutting concerns takes place starting from the use case models. A variety of approaches have been proposed in literature $[3,13]$ that can be employed for this activity. Once the identification of crosscutting concerns is considered to be finished, the use cases are reengineered so that each use case is affected by at most one concern. The Use Case 
Approach allows to model this separation as an extension use case that is connected by an extend-relationship to an extension point in the extended (base) use case.

Activities 1 and 2 are comparable to existing proposals and practices. The subsequent activities $(3,4)$ are essential and specific to our approach. The reengineering effort, from activity 2 , typically results in highly coupled use case models, where the implicit relations between an extension use case and the multiple base use cases it affects, are made explicit. This explicitation is done for each extension use case. The goal of activity 3 is to generalize these use case models in order to reduce coupling. It results in multiple use case hierarchies of which the roots are abstract use cases with generalized extension points. These generalized extension points form the first indication for finding stable domain abstractions for crosscutting concern composition. Selecting a name for a generalized extension point involves paying ample attention to finding the domain abstraction that best describes the semantics of the extension point in question. The guiding principle is that the domain abstractions should be anchored in the domain model.

Activity 4 is the final requirements engineering activity in our approach. During this activity, the generalized extension points are used to define domain abstractions. This definition contains a name and references to other domain concepts that are required for the particular composition-context: the contextual information.

In activity 5 , the first architectural design activity, the architecture is created (in fact the component-and-connector view) with the earlier-defined domain abstractions in mind. These domain abstractions constrain the design of software components and their interfaces to ensure that these artifacts remain compatible with the domain abstractions. This contrasts with the AO Use Case approach of Jacobson, et al., where a base architecture is defined without taking crosscutting concerns into account. In our approach, the domain abstractions ensure that the architectural design takes crosscutting concerns into account from its initial inception.

The previous activity, ensuring compatibility between domain abstractions and architecture, is important for the next activity (activity 6), where domain abstractions are mapped to pointcut interfaces. A pointcut interface has a scope that is limited to a module in the architecture (typically component, or a set thereof). It captures the artifacts in this module that match the specific domain abstraction; i.e. the named abstraction and the contextual information. In case a mismatch between an abstraction and the initial architecture prohibits the correct specification of this pointcut interface, the previous activity (activity 5 ) has to be repeated. Once the abstraction mismatch is corrected, the pointcut interface specification can be completed (repeat of activity 6 ).

In the final architecture design activity (activity 7), aspectual compositions are specified in terms of the pointcut interfaces, leveraging upon state-of-the-art aspect-oriented architectural description languages (ADL's).

\section{DETAILED DESCRIPTION AND ILLUS- TRATION}

This section presents a detailed explanation of the seven activities of the approach. At the same time, these different activities are illustrated in a running example. The final paragraph presents a closer look at the activities at the transition from requirements to architecture.

\subsection{Define Domain Model and Use Cases}

Throughout this section, the E-Finance Banking System [21, 23] serves as running example. Although somewhat pedagogical in na-

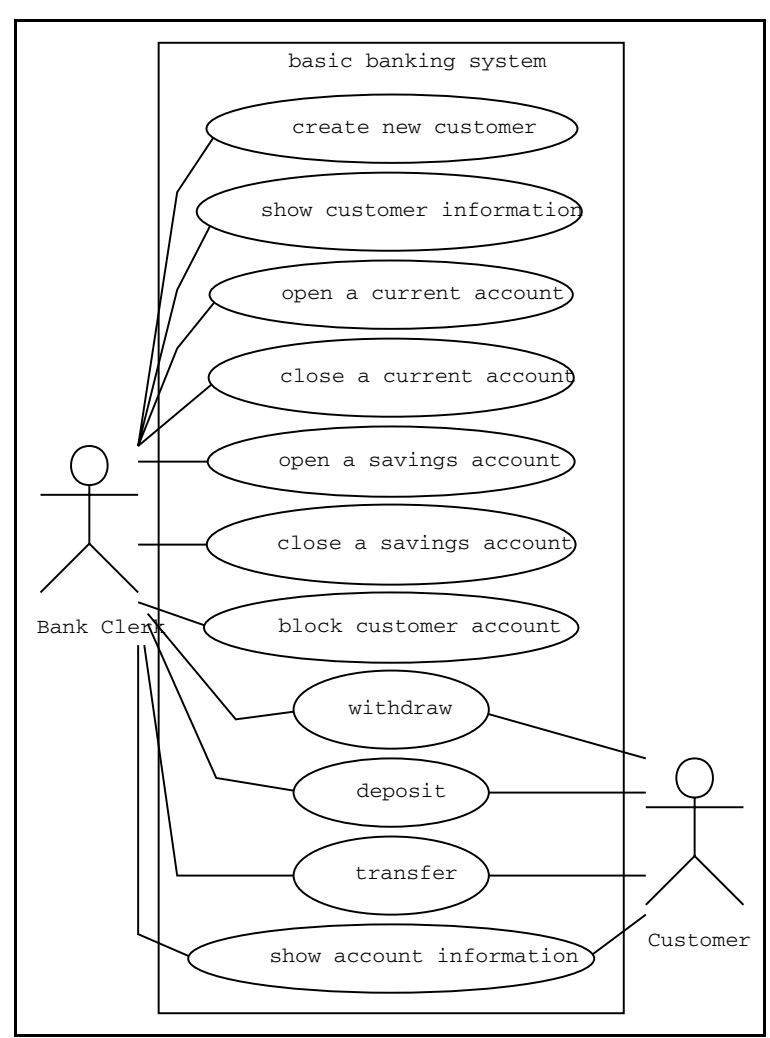

Figure 3: Use case diagram providing an overview of the basic banking services.

Use Case 1 Transfer between two accounts

- Primary actor: Bank clerk or Customer

\section{- Basic flow:}

1. The actor indicates he wants to transfer money.

2. The system asks for the source account.

3. The actor selects the source account.

4. The system asks for the destination account.

5. The actor enters the destination account.

6. The system asks for the amount to transfer.

7. The actor enters the amount.

8. The system verifies the balance of the source account.

9. The system asks to sign the transfer.

10. The actor signs the transfer.

11. The system checks the signature for validity.

12. The system processes the transfer.

\section{- Alternative flow:}

$8 \mathrm{~b}$ If the account balance is insufficient, the transaction is canceled.

$11 \mathrm{~b}$ If the provided signature is invalid, the transaction is canceled. 
ture, it covers a sufficiently large requirements space to be representative for a realistic application.

Activity 1 consists of diverse requirements gathering and engineering efforts such as interviewing the stakeholders, investigating current state-of-the-art and -practice in the field, taking into account several (legal, technical) constraints, detailed domain analysis with domain experts, and so on.

For the E-Finance system, the result of activity 1 consists of (i) more than fifty coarse-grained use cases (functional requirements), (ii) non-functional requirements such as security, performance and availability, described in quality scenarios [6], and (iii) the domain models resulting from domain analysis.

Figure 3 presents a subset of the basic banking use cases. In these use cases two distinct actors interact with the system: the bank clerk and the customer. As part of the basic banking services, the bank clerk is given the ability to create customer accounts and inspect customer information. The customer in turn is allowed to open and close accounts via the bank clerk. Both actors are able to perform financial transactions such as money transfer, withdrawal, and deposit.

In Use Case 1, a trimmed version of the transfer between two accounts use case is presented. This use case is used as running example throughout the paper.

Figure 4 presents a subset of the domain model for the E-Finance case study. A domain model represents a conceptual model which describes the various entities involved in the system and their relationships.

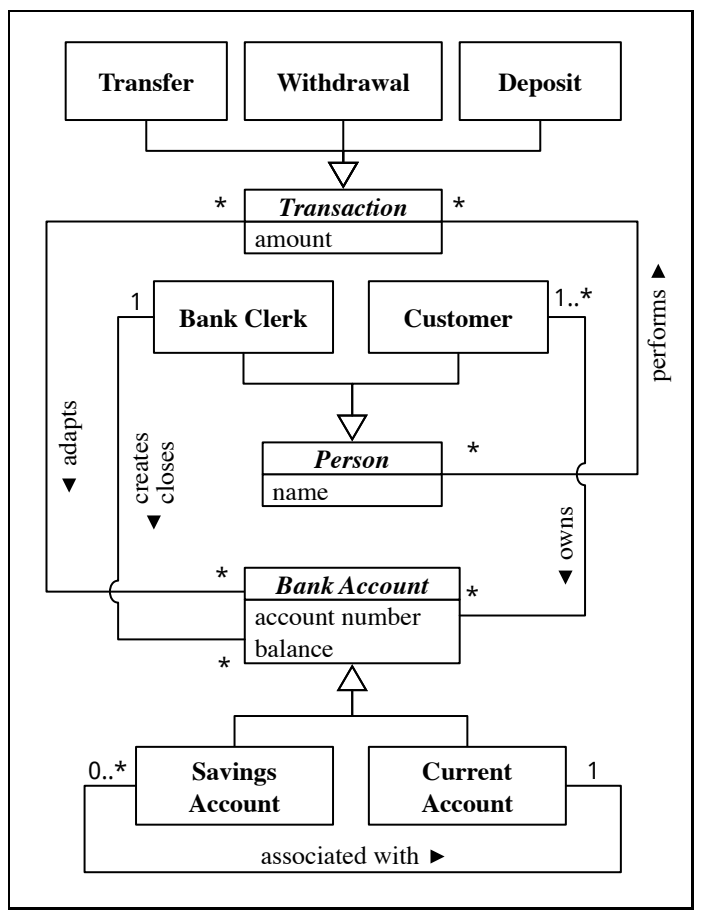

Figure 4: Subset of the E-Finance domain models.

\subsection{Identify and separate crosscutting con- cerns}

In the second activity, crosscutting concerns are identified in the use cases from previous activity. Thereafter, these use cases are reorganized, so that each use case is affected by at most one concern, a process called separation.
Identification of crosscutting concerns.

This sub-activity involves the identification of crosscutting concerns; i.e. concerns that affect several use cases. In use cases, crosscutting concerns can be identified by: (i) looking for recurring or duplicated steps across use cases, or (ii) paying specific attention to inclusion or extension relationships between use cases, as they represent interdependencies. As already stated in Section 3, existing aspect-oriented use case approaches can be adopted for implementing this identification process.

In the E-Finance use cases, the following crosscutting concerns were identified:

Balance verification. This concern handles verification that the balance of a bank account complies a certain constraint before executing a transaction. This business logic is triggered both at the closure of current and savings accounts to check if the final balance is zero, and at deposit, withdrawal and money transfer between accounts to check if the current balance is sufficient. In the example of Use Case 1, step 8 and its alternative step 8b, both belong to this crosscutting concern.

Agreement signing. When executing certain actions, the main actor is asked to confirm by signing a legally binding agreement with the bank. This happens when current and savings accounts are opened and closed, when the bank clerk blocks an account and when money is deposited, transferred, and withdrawn. In the example of Use Case 1, steps 9 to 11, as well as step $11 \mathrm{~b}$ are specific to this concern.

\section{Separation of crosscutting concerns.}

Next, the use cases are reorganized so that each use case describes at most one concern. A new extension use case is introduced for each set of recurring or duplicated use case steps. Next, these extension use cases are related back to the base use cases by means of the extend relation. In Use Case 2, the result of separating the crosscutting concerns in Use Case 1 is presented. During this process, one extension point is introduced pointing to step 8, as this is exactly the step at which the system must process the money transfer. For that reason, it is named money transfer. At this extension point, the use case is extended by the extension use case sign an agreement, which is presented in Use Case 3.

The full use case diagram after separating crosscutting concerns is presented in Figure 5. This diagram depicts both the base use cases and the extension use cases - sign an agreement (dark gray) and verify account balance (light gray) - together with the extension relations between them.

Use Case 2 Transfer between two accounts

- Primary actor: Bank clerk or Customer

- Basic flow:

1. The actor indicates he wants to transfer money.

2. The system asks for the source account.

3. The actor selects the source account.

4. The system asks for the destination account

5. The actor enters the destination account

6. The system asks for the amount to transfer.

7. The actor enters the amount.

8. Extension point: money transfer. The system processes the transfer. 
Use Case 3 Sign an agreement

- Primary actor: Bank clerk or Customer

- Extends:

- open a current account at open current account.

- close a current account at close current account.

- open a savings account at open savings account.

- open a savings account at close savings account.

- block customer account at block account.

- withdraw at money withdrawal.

- deposit at money deposit.

- transfer at money transfer.

- Basic flow:

1. The system asks to sign an agreement.

2. The main actor signs the agreement.

3. The system checks the provided signature for validity.

- Alternative flow:

$3 \mathrm{~b}$ If the provided signature is invalid, the use case has failed.

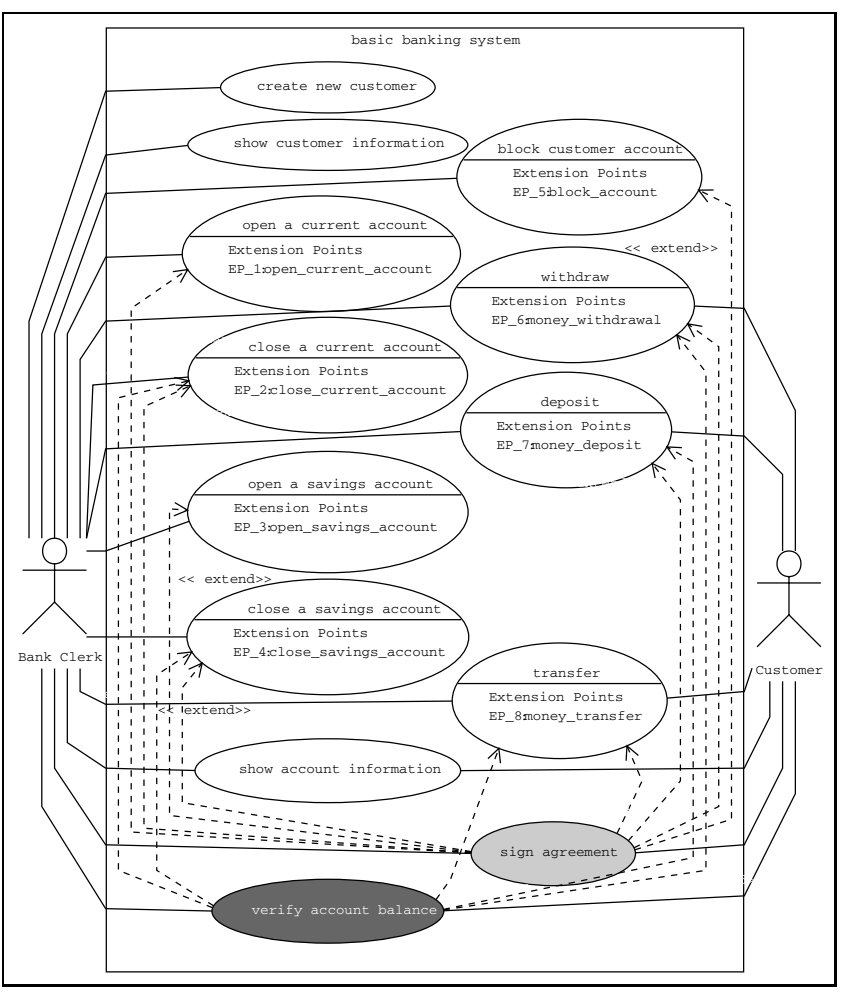

Figure 5: Use case diagram providing an overview of the basic banking services after separating the crosscutting concerns.

\subsection{Generalize crosscutting use case extend- relationships}

After previous activity, the crosscutting concerns are represented modularly (see Figure 5). However, the increased modularity clearly comes at the cost of maintainability and reusability. More specifically, a large set of extension points is introduced - one per base use case - at which the extension use cases extend the base. As a result, a high degree of coupling between base and extension use cases is introduced.

This complexity originates from the requirements themselves: it manifests itself differently depending on the particular modularization that was chosen, yet it inherently is problem space complexity. Therefore, removing this complexity is not the goal, managing it more efficiently, however, is. More specifically, this approach enables to better manage the complexity through the introduction of reusable abstractions.

To discover abstractions suitable for this purpose, the base use cases are placed in a generalization hierarchy. This leads to the introduction of several abstract use cases. This process is driven by the search for commonalities in the base use cases. For the use cases extended by the sign an agreement extension use case, the commonality is that they all adapt the customer's bank accounts (creation, closure, money transfer, etc). As a result, a specialization hierarchy is introduced, with from top to bottom: the abstract adapt an account use case (Use Case 4), the abstract perform a transaction use case (Use Case 5) and the transfer between two accounts use case (Use Case 6).

Use Case 4 [abstract use case] Adapt an account

- Primary actor: Bank clerk or Customer

\section{- Basic flow:}

1. [abstract] The actor indicates he wants to adapt an account.

2. The system asks for the account to adapt.

3. The actor selects an account.

4. [abstract] The system asks for the additional information.

5. [abstract] The actor enters the additional information.

6. Extension point: account adaptation. [abstract] The system performs the adaptation.

Use Case 5 [abstract use case] Perform a transaction

- Specializes: Adapt an account (Use Case 4)

- Primary actor: Bank clerk or Customer

- Basic flow:

1. [abstract] refines parent step 1 . The actor indicates he wants to perform a transaction.

2. Specialize step 2 The system asks for the source account.

3. As parent step 3.

4. As parent step 4 .

5. As parent step 5.

6. Extension point: balance change. [abstract] The system processes the transaction.

In Use Case 7, the extension use case sign an agreement extends the abstract use case adapt an account at the newly-introduced extension point account adaptation. Similarly for the balance veri- 
Use Case 6 Transfer between two accounts

- Specializes: Perform a transaction (Use Case 5)

- Primary actor: Bank clerk or Customer

- Basic flow:

1. Impl. step 1. The actor indicates he wants to transfer money.

2. As parent step 2 .

3. As parent step 3 .

4. Impl. step 4. The system asks for the destination account.

5. Impl. step 5. The actor enters the destination account

6. Impl. step 5. The system asks for the amount to transfer.

7. Impl. step 5. The actor enters the amount.

8. Impl. step 6 . The system processes the money transfer.

Use Case 7 Sign an agreement.

- Primary actor: Bank clerk or Customer

- Extends:

- adapt an account at account adaptation.

- Basic flow:

1. The system asks to sign an agreement.

2. The actor signs the agreement.

3. The system checks the provided signature for validity.

- Alternative flow:

3b If the provided signature is invalid, the use case has failed.

fication concern, the use cases to perform a transaction, open and close an account are grouped together, albeit deeper in the inheritance hierarchy. Figure 6 presents the resulting use case diagram.

Comparing this diagram to Figure 5, it becomes clear that the high coupling between base and extension use cases is represented in a more maintainable manner. Furthermore, the extension use cases are coupled to abstract concepts rooted in the problem domain.

\subsection{Define domain abstractions}

The main result of the previous activities is the identification of commonalities in the base use cases, represented by abstract extension points. However, at this moment an extension point is merely a label to a (set of) location(s) in a use case. In this activity, stable domain abstractions are defined from these extension points. The key characteristic of these definitions is that they are fundamentally anchored in the domain model, i.e. they are presented in terms of key concepts in the problem domain.

A domain abstraction is defined by name, textual description, contextual information, and potential relations to other domain abstractions.

Contextual information is additional information associated to a domain abstraction, which is extracted from the domain model as well. One way to discover it is by explicitly specifying the composition between base and extension use cases, using a use case pointcut language such as AspectU [31]. This involves specifying use case-level pointcuts and employing context passing mechanisms between the use cases to explicitize which type of information must be accessible to the extension use case. Due to space constraints, we do not elaborate on this further. In the case of the running example, the contextual information associated to the $\boldsymbol{a c}$ count adaptation domain abstraction consists of the bank account,

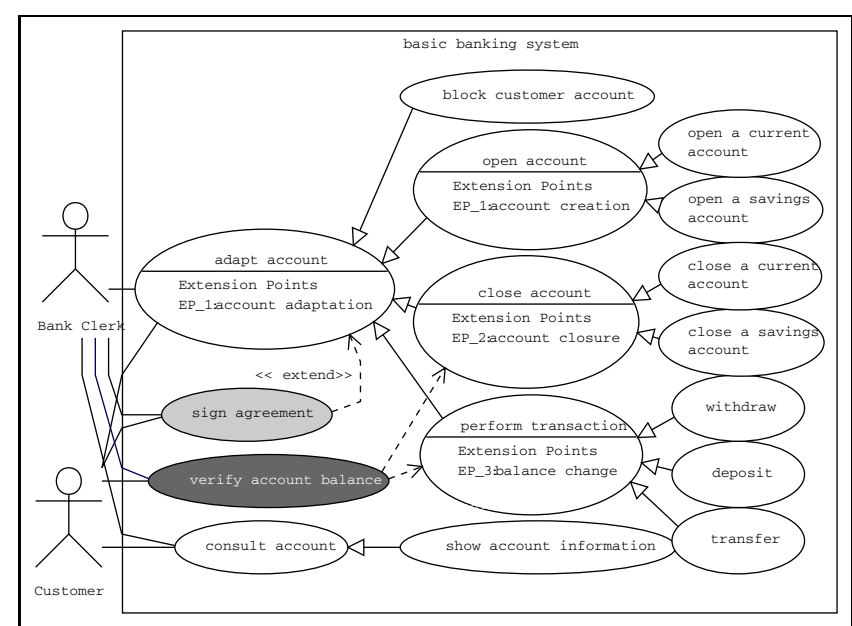

Figure 6: Use case diagram providing an overview of the basic banking services after the generalization step.

\section{account adaptation}

- Specialized by: account closure, account creation, balance change.

- Description: This domain abstraction is used to denote an adaptation to a bank account.

- Contextual information: the person that performs the adaptation (either the bank clerk or the customer), a reference to the bank account that is closed, and the bank account type.

Figure 7: The definition of the account adaptation domain abstraction. Underlined words denote contextual information.

the bank account type, and the person performing the adaptation (either a bank clerk or a customer), as presented in Figure 7.

All domain abstractions are placed into a domain abstraction inventory which is considered an integral part of the requirements documentation. In further activities (see Section 4.6), the domain abstraction inventory is used to specify pointcut interfaces in a concrete software architecture.

\subsection{Architectural design using domain ab- stractions}

This activity either starts with a pre-existing initial architecture, or with the design of an architecture. For this, a state-of-the-art software architecture methodology is employed, such as AttributeDriven Design [6]. The core activity in software architecture design is the introduction of a decomposition; i.e. dividing the system in software modules such as classes or software components. The end result is a software architecture that fullfills all requirements - functional and non-functional — and covers the problem domain.

Our approach extends this by constraining the software architect during architectural design so that the domain abstractions can in fact be implemented. In some cases, this implies that the architect must explicitly expose additional context information through the component interfaces.

Also, to ensure correctness of the compositions between aspect and base modules, it is during this activity that design rules [34, 19] would be defined by the software architect. However, these are out of scope of this paper. 


\subsection{Specify pointcut interfaces}

During this activity, the domain abstractions are mapped to pointcut interfaces. To achieve this, the used architecture description language (ADL) must support aspect-oriented compositions. Therefore, an aspect-oriented architecture approach is adopted. In this paper, we employ the AO-ADL [29].

As an alternative to the classical component interface, the pointcut interface is introduced. Indeed, not unlike the classical interface, the pointcut interface exposes the component's internals to the outside world. The representation we adopted for this is the UML lollipop stereotyped with pointcut interface (in short p.i.). This representation is shown in Figure 8. Note that a pointcut interface can be associated to any classifier (class, package, ...). However, due to our choice for a component-based architecture methodology in the running example, the pointcut interface will be associated with software components.

The specification of a pointcut interface comprises a set of named pointcuts. The pointcut parameters originate directly from the contextual information associated to the domain abstraction. For the running example, we have adopted the AspectJ syntax to specify the named pointcut in architecture. The pointcut interface is presented in Listing 1 . The pointcut signature matches exactly the contextual parameters of the account closure domain abstraction that was presented in Figure 7.

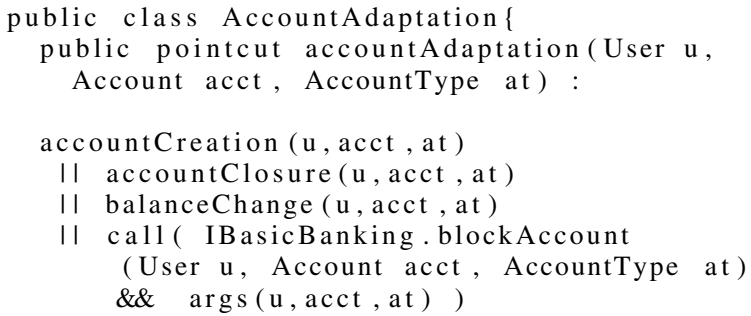

Listing 1: Definition of the account adaptation pointcut interface

During this activity, it might occur that the initial architecture fails to expose sufficient information to specify the pointcut interface correctly. This phenomenon is called abstraction mismatch. As a result, it may be required to repeat the architectural design phase, as part of activity 5 (see Section 4.5).

\subsection{Compose aspects through pointcut inter- faces}

Finally, the pointcut interfaces are used to define compositions. In the E-Finance case study, we have used the AO-ADL [29] to specify AO compositions. The idea is that there is no specialized component element for representing aspectual behaviour, however, there is a specific aspect-oriented connector for representing aspect-oriented composition logic as first-class entity. This is not a mandatory choice, as other aspect-oriented architecture techniques could be employed as well. This is elaborated further in Section 6.

Figure 8 presents an excerpt of the component-and-connector view of the resulting $\mathrm{AO}$ architecture. It illustrates one $\mathrm{AO}$ connectors, named sign agreement. It binds the pointcut interface account adaptation to the User Agreement Manager, responsible for creating an agreement and requesting the user to provide a signature. An AspectJ notation for this connector is presented in Listing 2.

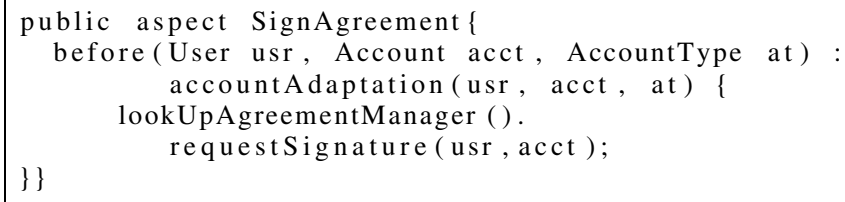

Listing 2: The Sign Agreement connector in AspectJ.

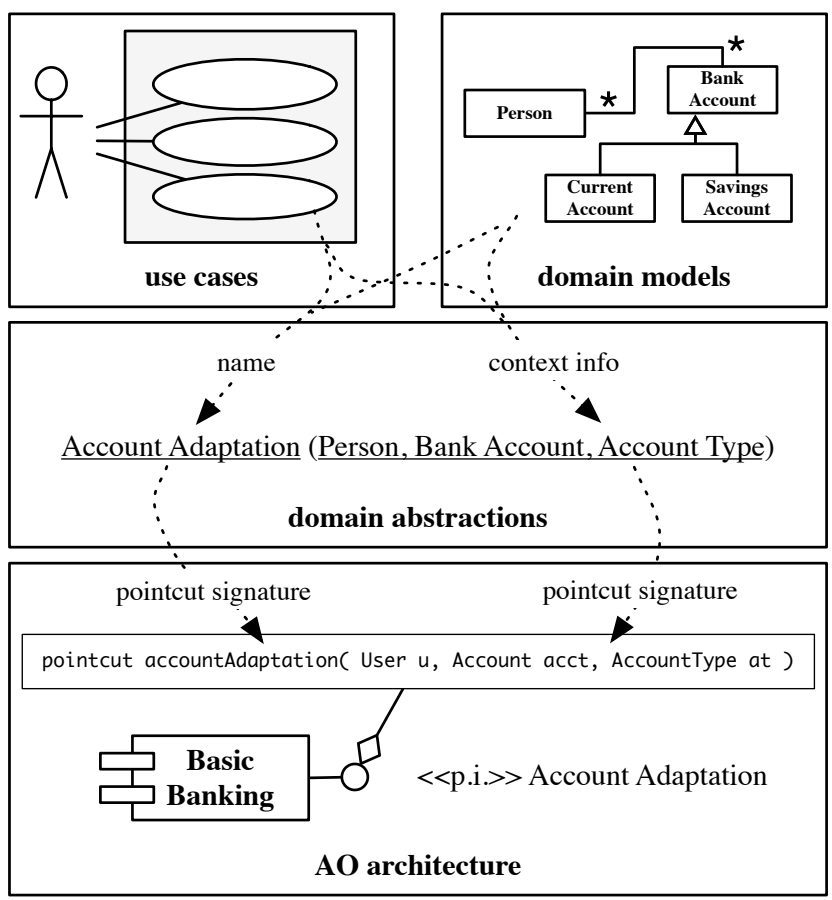

Figure 9: The transition from requirements to architecture.

\subsection{Transition from requirements to architec- ture}

This section provides a detailed zoom-in at the transition from requirements to architecture. We present an operational view of this transition process by giving a procedure of how to perform the activities involved (activities 4 to 6). Figure 9 illustrates the main artifacts involved in these activities. At the top of this figure, the use case diagram (left) represents the set of use cases that result from activity 3 , and the domain model (right) represents the domain models that are created in activity 1.

Activity 4: for each abstract extension point in the base use case hierarchy (as defined in activity 3 ):

- Specify and analyze the required data flow between the base use case and the extension use cases at the extension point (e.g. by using a use-case level pointcut language).

- Derive the required context information from the resulting data flow requirements.

- Create a domain abstraction which is named after the abstract extension point, and is related to the context information derived in the previous step.

The resulting domain abstraction specification is presented in the middle of Figure 9. 


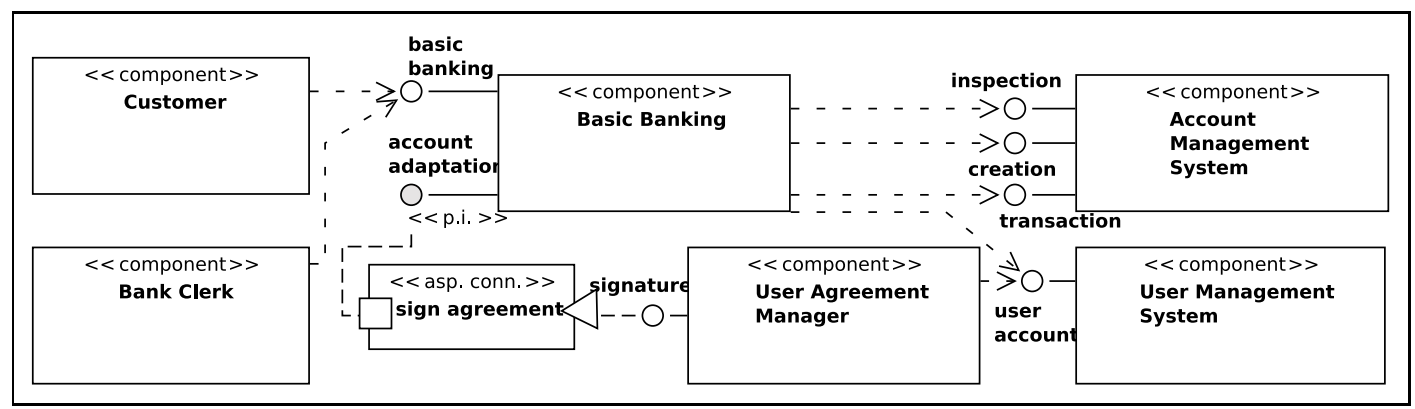

Figure 8: Subset of architecture for the E-Finance basic banking services.

Activity 5: either start from an existing architecture, or create an initial architecture using a state-of-the-art architecture method. During this activity, for each domain abstraction:

- Verify whether the current set of component interfaces exposes the required contextual information.

- If not, extend or modify the existing component interfaces to do so.

Activity 6: for each domain abstraction:

- Create a pointcut interface consisting of a poincut named after the domain abstraction. Add pointcut parameters to match the contextual information associated to the domain abstraction.

- If the architecture does not expose sufficient information (abstraction mismatch), go back to activity 5 (see Figure 2).

- Add the resulting pointcut interface to the suitable component(s).

The resulting pointcut interface is presented at the bottom of Figure 9.

\section{EXPERIENCE WITH THE METHOD}

We have applied the approach in two distinct case studies in which we evaluate how many pointcuts are effectively reusable over the course of system evolution. In both cases, we start from an existing development iteration in which the approach is applied to discover domain-anchored pointcut interfaces. Then, a second development iteration is adopted, introducing a substantial set of additional requirements, and the approach is re-applied. Finally, we assess effective reuse, clearly distinguishing between the three types of reuse as introduced earlier in Section 1.

E-Finance. This case study has been introduced in Section 4. A second development iteration has been performed, introducing new use cases into the case study adding investment services to the already-existing basic banking services.

Digital Publishing. Next to the E-Finance case study, we have also applied the approach to develop a digital news publishing platform [22]. Different from the E-Finance case, this case study starts from an existing component-based object-oriented system, which is re-factored using our approach to discover suitable pointcut interfaces. Then, a second development iteration is adopted, in which requirements are added. In this case, the evolution scenario represents a fundamental shift in the news publishing domain: starting from a classical news broadcasting (one-to-many) scheme, the system is adapted to support point-casting (a one-to-one scheme), by personalizing news editions to the end user's preferences.

Table 2 presents some facts and figures about both case studies. The first part of the table presents some figures to indicate their size and complexity. The second part of the table shows the relative
Table 2: Main facts and figures from case studies.

\begin{tabular}{|l|cc|cc|}
\hline Case study & \multicolumn{2}{|c|}{ E-Finance } & \multicolumn{2}{c|}{ Dig. Publishing } \\
Iteration & I & II & I & II \\
\hline \# use cases & 13 & 28 & 16 & 31 \\
\# crosscutting concerns & 2 & 4 & 3 & 7 \\
\# domain abstractions & 4 & 6 & 7 & 12 \\
\hline Total person months & \multicolumn{2}{|c|}{3} & & 4.5 \\
Person hours applying & \multicolumn{2}{|c|}{55} & & 116 \\
the approach & & & \\
\hline
\end{tabular}

amount of time needed to apply the approach in these case studies ${ }^{1}$. More information about both case studies can be found at [22, 23].

\section{Summary of Observations.}

\section{Reuse of existing pointcut interfaces.}

This type of reuse occurs when a new aspect is introduced that can be composed with the application in terms of already-existing pointcut interfaces, either fully or partially.

In the E-Finance case study, two crosscutting concerns were added: age verification and fraud detection. We were able to compose all aspects to the base by uniquely relying on the earlierdefined pointcut interfaces. One example is the age verification concern, which dictates that whenever an account is opened, the age of the legal holder has to be verified. In this example we were able to effectively reuse the account creation pointcut interface, exactly as it was defined in the first development iteration.

In the digital publishing case study, four system-level crosscutting concerns were added. For two of these, we were able to entirely specify the composition in terms of pointcut interfaces that were defined in the first development iteration. Interestingly, in the cases where we were forced to introduce new pointcut interfaces for aspect composition, the newly introduced domain abstractions were unrelated to the already-existing domain abstractions.

\section{Reuse of aspect compositions.}

This type of reuse occurs when a new requirement is added that affects an already-existing crosscutting concern. Reuse is successful when the new requirement can be supported by re-implementing an existing pointcut interface, without breaking the pointcut signatures. The re-implementation typically involves expanding the pointcuts to a larger join point set in the base application.

This type of reuse was most prominent in the E-Finance case study. For example, the new investment services introduced a new account type: the custody account. Because of this, the actual implementation of the account closure pointcut interface was adapted, without needing to break the pointcut signature. 
In the digital publishing system, we experienced this type of reuse as well, albeit to a lesser degree. For example, in the first iteration, a pointcut interface named user-system interaction was introduced. This pointcut interface matches to the set of operations callable by an untrusted user. In the first iteration, is was used to compose the authentication aspect to these operations. In the second iteration, the number of methods callable by untrusted users increased drastically. Triggered by this evolution, the implementation of this pointcut interface was adapted, but its signature was not broken.

\section{Reuse within pointcut interface hierarchies.}

This type of reuse occurs when the pointcut interfaces themselves are structured hierarchically; i.e. the implementation of highlevel pointcut interfaces is done in terms of lower-level ones. This structuring avoids unnecessary duplication of pointcut interface specifications, and whenever the implementation of a lower-level pointcut changes, the higher-level pointcut reuses this change at no extra cost.

In the E-Finance case study, this type of reuse was encountered in the account adaptation pointcut interface. As presented earlier (cf. Listing 1), this pointcut interface is an aggregate of the account closure, account creation, and balance change pointcut interfaces, as well as a concrete pointcut expression. Whenever the implementation of one of these lower-level pointcut interfaces changes, but not its signature, that change does not ripple through to the higherlevel pointcut interface account adaptation.

In the digital publishing system, the approach did not deliver a hierarchy of pointcut interfaces, and thus this type of reuse can not be claimed.

\section{Discussion.}

In summary, we effectively reused some of the pointcut interfaces discovered by our method in both case studies. More specifically, in the E-Finance case study, we effectively reused all four pointcut interfaces. In the digital publishing case study, we reused three out of six discovered pointcut interfaces.

To understand why this reuse generally was less prominent in the digital publishing case study, we have to consider the stability of both domains. As explained in Section 2, this is an important factor influencing the effectiveness of our approach. In both case studies, we were forced to change the domain models in the second iteration. However, a distinction has to be made between complementary changes, and invasive changes to the domain model. Examples of complementary changes are adding concepts as leaves of existing generalization/specialization hierarchies, adding concepts that have no intrinsic relation to earlier-defined concepts. Invasive changes in the domain are for example restructuring existing relationships between concepts, invasively redefining concepts, or removing concepts. In the E-Finance case study, the adaptations to the domain model were mostly complementary in nature. To illustrate, we found that the newly-introduced custody account fit well into the existing domain model, as it already contained the concept of a bank account (see Figure 4). On the other hand, the digital publishing evolution scenario brought more invasive changes to the publishing domain models. This was mostly due to the fact that the adopted evolution scenario represents a fundamental shift in digital news publishing. From these considerations, it becomes clear that the E-Finance domain was closer to the theoretical extreme of absolute domain stability than the digital publishing domain. This in turn provides an explanation why we indeed experienced a higher degree of effective reuse in the E-Finance case study.

Concluding, we state that in both case studies, (i) we experienced effective reuse of the pointcut interfaces introduced by our approach, and (ii) we found that the effectiveness of the presented approach to discover reusable pointcut interfaces is dependent on the stability of the domain it acts upon.

Since we applied this validation in only two case studies, it has no statistical relevance as such. A statistically relevant validation would include the application of the approach in multiple case studies, and application domains. Moreover, a full-blown validation of the approach would take into account other considerations such as the scalability of the approach, and the maintainability of the resulting software architectures. Providing such a strengthened validation of the approach is part of future work.

However, we clearly provide some compelling examples that illustrate effective reuse of the pointcut interfaces in an $\mathrm{AO}$ architecture as a result of applying the approach.

\section{RELATED WORK}

In this section we compare our domain-driven architecture method against four bodies of work: Early Aspects approaches, aspect-oriented architecture approaches, aspect-oriented approaches to use case modeling, and approaches that deal with the fragile pointcut problem.

\section{Early Aspects.}

As our domain-driven architecture method is situated at the transition between requirements and architecture, it is related to various Early Aspects [4] approaches that map aspectual requirements to architecture. The most relevant approaches in this space include COMPASS [7], Goal-Driven Discovery of Aspects [27], Theme [8], Jacobson's AOSD with Use Cases approach [15] and Aspect-Oriented Architecture Models (AAM) [10]. We first discuss the general relationship between these approaches and our domain-driven architecture method. Thereafter we more closely compare against COMPASS and the Goal-Driven Discovery approach as these approaches derive pointcut-like compositions from aspectual requirements.

The common goal of all the above Early Aspects approaches is achieving improved aspect traceability across the software life cycle and supporting trade-off analysis [30, 27] for conflicting requirements. Our domain-driven architecture method is complementary to these approaches as we focus on discovering stable architectural abstractions for aspect-oriented composition, starting from the requirements level. As our method adheres to the Twin Peaks model, the method should ideally be performed after a first iteration of these Early Aspects approaches has been performed.

In COMPASS, aspectual compositions at the requirements level are mapped to aspect-oriented compositions at the architecture level. More specifically, COMPASS uses a one-to-one mapping between requirements-level and architecture-level compositions. In contrast, our method takes a many-to-one mapping approach, where a single pointcut signature at the architectural level is derived by generalizing multiple aspectual compositions at the requirements level. This generalization step is the key factor that enables a higher reusability of pointcuts across multiple aspects. COMPASS also uses the AO-ADL architectural description language [29] for expressing aspectual compositions at the architecture level. AO connector templates are an interesting feature of AO-ADL which enable parametrization reuse of pointcuts. This kind of pointcut reuse is focused at reusing the binding of specific aspectual components across multiple base applications. In contrast, our method is focused at reusing a pointcut across multiple aspects.

The Goal-driven Discovery of Aspects approach offers detailed procedures for discovering aspects from V-shaped goal graphs [38]. 
These goal aspects are represented as pairs of pointcut-advice constructs, where advice refers to softgoals, and a pointcut refers to fine-grained functional goals. We believe our approach complements this by discovering stable domain abstractions in the goal aspects. Once these stable abstractions are identified, however, their projection to the operationalizations of the functional goals potentially leads to reusable pointcuts at the architecture level.

\section{Aspect-oriented architecture approaches.}

In the area of aspect-oriented product-line architectures [11,36], there are some works $[16,26,5,20]$ that focus on establishing stable aspectual interfaces, with the possibility of specifying various types of contracts to govern interactions between features (aspects) and the base architecture. As stated in section 4.5, specifying such interaction contracts and other design rules is possible, yet out of the scope of our method. Kulesza, et al. [20] also identified a synergy between extension points in use cases and aspectual interfaces, which they aim to exploit in future work to improve the traceability between requirements and architecture. In opposition, our approach exploits this synergy to discover stable abstractions for reusable aspectual interfaces.

\section{Aspect-oriented approaches to use case modeling.}

Most existing aspectual use-case approaches [2, 31, 13, 33] propose an explicit crosscuts relationship so that the crosscutting requirements can be completely modularized without having to prepare the base use cases with extension points. Our goal is not to achieve improved modularization of aspectual use cases. Instead we want to generalize base use cases to reveal stable domain abstractions for multiple aspectual use cases.

\section{Fragile pointcut problem.}

Most of the recent work on explicit pointcut interfaces at the implementation level, a concept originally proposed by Gudmundson and Kiczales [12], has to do with the fragile pointcut problem [18]. This problem refers to the situation when pointcuts suffer from unintended join point matches or accidental join point misses after base code has evolved [35]. The XPI approach [34] deals with this problem by attaching design rules to pointcut interfaces. These design rules are contract-like invariants about the structure of the base code, which can be automatically maintained over the course of system evolution. Open modules [1] deals with the problem by making the base code modules directly responsible for maintaining the implementation of the pointcut interfaces. The main contribution of these works thus lies in the fact that the correctness of pointcut interface implementations can be maintained in the face of changes in the base code. However, these works define very little guidance on how to identify the right abstractions so that a pointcut interface becomes useful for as many client aspects as possible. Our domain-driven method aims to provide such guidance, thus mostly making a complementary contribution at the client-side of the pointcut interface.

The work on model-based pointcuts [17] tackles the fragile pointcut problem by declaring pointcuts in terms of a conceptual model of the base program. The definition of the conceptual model is based on the Intentional Views approach [25]. Intentional views describe concepts of interest to a programmer by grouping program entities (classes, methods, ...) that share some structural property. For example, in an object-oriented implementation of a Wiki framework, the WikiAction view groups all methods implementing an action on a Wiki page. The question arises however whether the concept of a wiki action is a suitable abstraction for expressing the required aspect compositions in the Wiki framework. Again, we believe our method provides the missing guidance on identifying what are the relevant properties to expose in such an Intentional view.

\section{CONCLUSION AND FUTURE WORK}

This paper presents a method to discover stable domain abstractions from use cases and domain models. Furthermore, it guides the software architect in mapping these domain abstractions to reusable pointcut interfaces, and constraining the software architecture in order to implement the pointcut interfaces. This approach builds on a certain degree of domain stability; i.e. the problem domain remains stable under system evolution. More specifically under this precondition, the discovered domain abstractions can be considered to be a ubiquitous vocabulary, as they represent a complete terminology for the stakeholders to communicate about the software solutions in terms of problem space concepts. Specifying pointcut interfaces based on such stable domain abstractions improves the effective reusability of these pointcut interfaces. We have applied the approach in two realistic case studies, in which we adopted an evolution scenario to assess how many pointcut interfaces could effectively be reused. From this, we have shown that we were indeed able to reuse the pointcut interfaces discovered by applying the approach. More importantly, we found that the effectiveness of the approach indeed depends on the stability of the domain it acts upon.

In future work, we will strengthen the validation of the approach as outlined in Section 5. Also, investigating how closely our approach is tied to the specific choice of use cases as requirements representation technique is part of future work. Possibly a similar approach could be articulated for different requirement representation techniques, such as problem frames, viewpoints or goals.

Meanwhile, we have presented an architecture method that leverages upon state-of-the-art research in AORE and on state-ofpractice in requirements specification (use cases). We believe that this is essential to integrate and adopt AOSD in all stages of the software life cycle.

\section{Acknowledgements}

This research is supported by European Commission FP6 Grant AOSD-Europe: European Network of Excellence on AOSD (IST2-004349), the Interuniversity Attraction Poles Programme Belgian State, Belgian Science Policy, by the Research Fund K.U.Leuven, and by an SBO project grant from the Flemish Institute for the advancement of scientific-technological research in industry (IWT).

\section{REFERENCES}

[1] Jonathan Aldrich. Open modules: Modular reasoning about advice. In ECOOP, pages 144-168, 2005.

[2] João Araújo, Ana Moreira, Isabel Brito, and Awais Rashid. Aspect-oriented requirements with UML. In Mohamed Kandé, Omar Aldawud, Grady Booch, and Bill Harrison, editors, Workshop on Aspect-Oriented Modeling with UML, 2002.

[3] João Araújo and Ana M. D. Moreira. An aspectual use-case driven approach. In Ernesto Pimentel, Nieves R. Brisaboa, and Jaime Gómez, editors, JISBD, pages 463-468, 2003.

[4] Elisa L. A. Baniassad, Paul C. Clements, João Araújo, Ana Moreira, Awais Rashid, and Bedir Tekinerdogan. Discovering early aspects. IEEE Software, 23(1):61-70, 2006.

[5] Olivier Barais, Eric Cariou, Laurence Duchien, Nicolas Pessemier, and Lionel Seinturier. Transat: A framework for 
the specification of software architecture evolution. In ECOOP First International Workshop on Coordination and Adaptation Techniques for Software Entities (WCAT04), Oslo, Norway, jun 2004.

[6] L. Bass, P. Clements, and R. Kazman. Software Architecture in Practice. Addison-Wesley, second edition, 2003.

[7] Ruzanna Chitchyan, Mónica Pinto, Awais Rashid, and Lidia Fuentes. Compass: Composition-centric mapping of aspectual requirements to architecture. Transactions on Aspect-Oriented Software Development IV, pages 3-53, 2007.

[8] S. Clarke and E. Baniassad. Aspect-Oriented Analysis and Design: the Theme Approach. Addison-Wesley Reading, 2005.

[9] Eric Evans. Domain-Driven Design: Tackling Complexity in the Heart of Software. Addison-Wesley, 2004.

[10] Robert B. France, Indrakshi Ray, Geri Georg, and Sudipto Ghosh. Aspect-oriented approach to early design modelling. IEE Proceedings - Software, 151(4):173-186, 2004.

[11] Martin L. Griss. Implementing product-line features by composing aspects. In Patrick Donohoe, editor, SPLC, pages 271-289. Kluwer, 2000.

[12] Stephan Gudmundson and Gregor Kiczales. Addressing practical software development issues in aspectj with a pointcut interface. In Advanced Separation of Concerns, 2001.

[13] Stephan Herrmann, Christine Hundt, and Katharina Mehner. Mapping use case level aspects to object teams/java. In OOPSLA Workshop on Early Aspects, 2004.

[14] Martin E. Nordberg III. Aspect-oriented dependency inversion. In Workshop on Advanced Separation of Concerns in Object-Oriented Systems at OOPSLA, 2001.

[15] Ivar Jacobson and Pan-Wei Ng. Aspect-Oriented Software Development with Use Cases. Addison-Wesley, 1st edition, December 2004.

[16] Mika Katara and Shmuel Katz. Architectural views of aspects. In AOSD '03: Proceedings of the 2nd international conference on Aspect-oriented software development, pages 1-10, New York, NY, USA, 2003. ACM.

[17] Andy Kellens, Kim Mens, Johan Brichau, and Kris Gybels. Managing the evolution of aspect-oriented software with model-based pointcuts. In Dave Thomas, editor, ECOOP, volume 4067 of Lecture Notes in Computer Science, pages 501-525. Springer, 2006.

[18] C. Koppen and M. Stoerzer. Pcdiff:attacking the fragile pointcut problem. In First European Interactive Workshop on Aspects in Software (EIWAS), 2004.

[19] Uirá Kulesza, Er Alves, Ro Garcia, Carlos J. P. De Lucena, and Paulo Borba. Improving extensibility of object-oriented frameworks with aspect-oriented programming. In Proc. of the 9th Intl Conf. on Software Reuse (ICSR'06, pages 231-245, 2006.

[20] Uirá Kulesza, Er Alves, Ro Garcia, Alberto Costa Neto, Elder Cirilo, Carlos J. P. De Lucena, and Paulo Borba. Mapping features to aspects: A model-based generative approach. In Early Aspects: Current Challenges and Future Directions, volume 4765 of LNCS. Springer, 2007.

[21] Bert Lagaisse, Bart De Win, Wouter Joosen, and Johan Van Oeyen. E-finance case study: analysis and requirements. CW-Report 438, DistriNet, KULeuven, March 2006.

[22] Dimitri Van Landuyt, Steven Op de beeck, Bas Kemper,
Eddy Truyen, and Wouter Joosen. Building a next-generation digital publishing platform using aosd; http://distrinet.cs.kuleuven.be/projects/digitalpublishing/.

[23] Dimitri Van Landuyt, Steven Op de beeck, Eddy Truyen, and Wouter Joosen. An aspect-oriented architecture for the e-finance case study; http://distrinet.cs.kuleuven.be/projects/aoarchitectureefinance/.

[24] Jacques Meekel, Thomas B. Horton, Robert B. France, Charlie Mellone, and Sajid Dalvi. From domain models to architecture frameworks. In SSR '97: Proceedings of the 1997 symposium on Software reusability, pages 75-80, New York, NY, USA, 1997. ACM.

[25] Kim Mens, Andy Kellens, Frédéric Pluquet, and Roel Wuyts. Co-evolving code and design with intentional views: A case study. Computer Languages, Systems \& Structures, 32(2-3):140-156, 2006.

[26] Freddy Munoz, Benoit Baudry, and Olivier Barais. Improving maintenance in aop through an interaction specification framework. In ICSM, pages 77-86. IEEE, 2008.

[27] Nan Niu and Steve Easterbrook. Analysis of early aspects in requirements goal models: A concept-driven approach. Transactions on Aspect-Oriented Software Development III, pages 40-72, 2007.

[28] Bashar Nuseibeh. Weaving together requirements and architectures. IEEE Computer, 34(3):115-117, 2001.

[29] M. Pinto and L. Fuentes. AO-ADL: An ADL for describing aspect-oriented architectures. In Early Aspects: Current Challenges and Future Directions, volume 4765 of LNCS. Springer, 2007.

[30] Awais Rashid, Ana M. D. Moreira, and João Araújo. Modularisation and composition of aspectual requirements. In $A O S D$, pages 11-20, 2003.

[31] Jonathan Sillito, Christopher Dutchyn, Andrew David Eisenberg, and Kris De Volder. Use case level pointcuts. In Martin Odersky, editor, ECOOP, volume 3086 of Lecture Notes in Computer Science, pages 244-266. Springer, 2004.

[32] Ian Sommerville. Software Engineering. Pearson, 2008.

[33] Geórgia Sousa, Sérgio Soares, Paulo Borba, and Jaelson Castro. Separation of crosscutting concerns from requirements to design: Adapting the use case driven approach. In In Proc. Early Aspects Workshop at AOSD, 2004.

[34] Kevin J. Sullivan, William G. Griswold, Yuanyuan Song, Yuanfang Cai, Macneil Shonle, Nishit Tewari, and Hridesh Rajan. Information hiding interfaces for aspect-oriented design. In Michel Wermelinger and Harald Gall, editors, ESEC/SIGSOFT FSE, pages 166-175. ACM, 2005.

[35] Tom Tourwé. On the existence of the aosd-evolution paradox. In In AOSD Workshop on Software Engineering Properties of Languages for Aspect Technologies, 2003.

[36] Markus Völter and Iris Groher. Product line implementation using aspect-oriented and model-driven software development. In SPLC, pages 233-242. IEEE Computer Society, 2007.

[37] Dean Wampler. Aspect-oriented design principles: Lessons from object-oriented design. In Proceedings of the 2007 AOSD conference. Object Mentor, Inc., Feb. 2007.

[38] Yijun Yu, Julio Cesar Sampaio do Prado Leite, and John Mylopoulos. From goals to aspects: Discovering aspects from requirements goal models. In $R E$, pages 38-47. IEEE Computer Society, 2004. 